\title{
Two-way coupled particle-laden mixing layer. Part 1: Linear instability
}

\author{
X.-L. Tong, L.-P. Wang* \\ Department of Mechanical Engineering, University of Delaware, Newark, Delaware 19716, USA
}

Received 20 May 1998; received in revised form 2 September 1998

\begin{abstract}
The two-way coupled particle-laden mixing layer is studied numerically and theoretically in order to understand how the addition of solid particles affects the stability of the gas flow. Numerical simulations are undertaken to obtain results equivalent to solving the Orr-Sommerfeld equation of a dusty gas mixing layer first derived by Saffman. The growth rate of a viscous particle-laden mixing layer depends on the wavenumber, flow Reynolds number, Stokes number, and bulk particulate mass loading. Two asymptotic relations proposed by Saffman have been confirmed for the first time by numerical simulations. In addition to the stabilizing effect of particles on the gas flow at large Stokes number, a destabilizing influence at small Stokes number is also observed at finite flow Reynolds number. The fact that the addition of particles can destabilize the gas flow in the absence of gravity has been shown to follow the original speculation of Saffman. Physically, the increase of effective inertia of the fluid-particle mixture causes a destabilization effect, while the enhanced viscous dissipation around particles gives a stabilization effect. These qualitatively different effects have been shown to be directly related to the direction of interphase energy transfer. Results at arbitrary mass loading, Stokes number, and wavenumber show that for a given mass loading and wavenumber, there is an intermediate Stokes number which corresponds to a maximum flow stability. We have shown that this Stokes number is on the order of one, and depends on the wavenumber. An analytical model for predicting the growth rate in a viscous, particle-laden gas mixing layer is proposed and compared with the simulation results. (C) 1999 Elsevier Science Ltd. All rights reserved.
\end{abstract}

Keywords: Particle-laden flow; Instability; Mixing layer; Two-way coupling; Stokes number; Mass loading

\section{Introduction}

Particle-laden two-phase flows are encountered in a wide range of industrial applications under various flow configurations. Most of the studies in the past have focused on the

\footnotetext{
*Corresponding author. Tel.: (302) 831-8160; fax: (302) 831-3619; lwang@me.udel.edu.
} 
turbulent dispersion of particles in dilute flows [e.g. see Shirolkar et al. (1996) for a recent review]. Moderate mass loadings of particles can also alter the fluid flow, which is known as the flow modulation (or turbulence modulation if the carrier flow is turbulent). In gas-solid flows, three qualitatively different flow regimes can be defined, depending on the level of particulate volume fraction and mass loading (Elghobashi 1994). First, at very low volume fractions and mass loadings, the particles do not affect the surrounding flow and there is no flow modulation. This is known as the one-way coupling regime. In the second regime, the particulate volume fraction is very low due to small particle sizes, but the mass loading is on the order of one because of the high particle to fluid density ratio. In this case, particles can significantly change the carrier-flow characteristics, but particle-particle interactions are often neglected. This is called the two-way coupling regime. Finally, in the dense flow regime, both the volume fraction and mass concentration are high so that particle-particle collisions have to be considered. In this study, we will address particle-laden mixing layers which are volumetrically dilute, but have a large mass loading (the second regime).

Turbulence or flow modulation by particles has been known for several decades. Early experimental observations (Torobin and Gauvin 1961) showed that the presence of particles changes the wall drag in pipes as well as rates of heat transfer and chemical reaction which cannot be explained unless the fluid turbulence is modified by particles. Since then, the issue of whether turbulence in the carrier flow is enhanced or reduced by the dispersed particles has been the subject of many experimental studies as experimental techniques have advanced. The survey by Gore and Crowe (1991) showed that a range of effects may be observed depending on the particle size and volumetric loading of particles. They found that the ratio of particle diameter to turbulence integral length is a good indicator of whether the fluid turbulence level is increased or decreased. Hetsroni (1989) included the particle inertia in his analysis and correlated the turbulence enhancement or suppression in terms of particle Reynolds number based on the interphase slip velocity and particle diameter. He concluded that the presence of particles with a low particle Reynolds number tends to decrease the fluid turbulence and particles with high Reynolds number tends to increase the fluid turbulence. Recently, several theoretical models have been developed to predict the direction and level of turbulence modification (Yuan and Michaelides 1992, Yarin and Hetsroni 1994, Crowe et al. 1997).

With the development of modern computers, direct numerical simulations provide an alternative tool for studying turbulence modulation by particles. For example, Squires and Eaton (1990) reported that the particles enhance the turbulent kinetic energy at high wavenumbers while decreasing the turbulent kinetic energy at low wavenumbers in forced isotropic, stationary turbulence. Elghobashi and Truesdell (1993) found a similar "pivoting" of the energy spectrum in a decaying, isotropic, particle-laden turbulent flow.

The problem of turbulence modulation in fully developed flows is related to the question of the stability and evolution of two-phase laminar flows. In this study, we investigate flow modulation in a particle-laden mixing layer, including the linear instability in this paper and nonlinear evolution in a companion paper (Wang et al. 1998a).

The mixing layer represents a simple shear flow and thus provides a building block for many practical, inhomogeneous flows such as jets, wakes and flow over objects. There have been numerous experimental and numerical studies of particle transport in mixing layers under oneway coupling (e.g. see Samimy and Lele 1991, Hishida et al. 1992, Lazaro and Lasheras 
1992a,b, Wang 1992, Crowe et al. 1993, Martin and Meiburg 1994, Raju and Meiburg 1995, Wang et al. 1998b). Particle transport in a mixing layer differs significantly from that of passive tracers if the particle inertial response time is comparable to the characteristic time scale of the flow. Particles are found to accumulate in the periphery of large-scale vortices and particularly near the braid stagnation points. The level of accumulation may be very high so that local particle concentration or mass loading may be much larger than the bulk mean value.

There are relatively few studies of two-way coupled, particle-laden mixing layers. Saffman (1962) presented the first analytical formulation on this subject. He derived a modified OrrSommerfeld equation to study the linear stability of viscous, particle-laden, plane parallel flows, under the assumptions of a dilute monodisperse suspension of fine particles and uniform initial particle concentration. The momentum coupling is handled by a force term proportional to the local interphase velocity slip and particle concentration. The presence of particles introduces two additional parameters to the stability problem, namely, the bulk mass loading and the particle Stokes number (the ratio of particle response time to flow characteristic time). Saffman concluded that the addition of very weak-inertia particles (i.e. very small Stokes number) tends to destabilize the flow, while the addition of very large-inertia particles stabilizes the flow. Michael (1965) investigated the Kelvin-Helmholtz instability of an inviscid dusty gas by applying a step mean flow velocity profile and showed that the presence of the particles always stabilizes the flow. Acrivos and co-workers (Herbolzheimer 1983, Shaqfeh and Acrivos 1986, Borhan and Acrivos 1988) systematically studied the stability of buoyancy-driven twophase interfacial flows in inclined particle settlers and found both inertia and buoyancy can cause the growth of instability waves at the interface. Yang et al. (1990) integrated numerically a modified Rayleigh instability equation for an inviscid, spatially-evolving gas-solid mixing layer. They neglected the particle perturbation velocity completely in their work and showed that the stability of the mixing layer is always enhanced with increased particle loading and decreased particle free-stream velocity ratio. Sykes and Lyell (1994) performed the linear spatial stability analysis of an inviscid, particle-laden circular jet under the similar assumptions of Yang et al. (1990) and reached the same conclusion that particles always stabilize the flow. Isakov and Rudnyak (1995) investigated the neutral stability curves of a dusty channel flow, showing similar trends of Saffman (1962) that small inertia particles destabilize the flow while large inertia particles stabilize the flow. Wen and Evans (1994) extended the work of Yang et al. (1990) by having non-uniform mean particle concentration in the shear layer. They observed the occurrence of two separate modes as a result of the non-uniform loading: a long wave mode which corresponds to the standard Kelvin-Helmholtz instability and a short wave mode which is similar to Holmboe instabilities observed in stratified flows. A more careful study of the spatial instability of an inviscid, particle-laden mixing layer is given by Dimas and Kiger (1998). They included the particle perturbation velocity so their results are applicable to finite particle inertia.

In this paper, numerical simulations are performed to study the linear instability of a viscous, two-dimensional, particle-laden mixing layer. We find that while for most of the parametric region the presence of particles stabilizes the flow, addition of weak-inertia particles can destabilize the flow even in the absence of a gravitational force field, in a manner consistent with Saffman's (1962) prediction. A relation is developed to predict the growth rate and compare this with numerical results. The paper is organized as follows. In Section 2 we 
present the mathematical formulation and theoretical predictions; the numerical method is described in Section 3; numerical results are discussed and compared with theories in Section 4, as well as an alternative interpretation in terms of the direction of interphase energy transfer.

\section{Theory}

We consider a gas mixing layer laden with solid particles in which the volume fraction of particulate phase is very low and particles are uniform in size with diameter much smaller than any characteristic length scales in the base gas flow. The particle density is much larger than that of the gas $\left(\rho_{\mathrm{p}} / \rho_{\mathrm{f}} \gg 1\right)$ so that the bulk mass loading of the particulate phase is on the order of one and as such the flow modulation by the particles may be significant. The dimensional governing equations for the carrier gas (subscript f) and particulate phase (subscript $\mathrm{p}$ ) are:

$$
\begin{aligned}
& \nabla \cdot \mathbf{u}=0 \\
& \frac{\partial \mathbf{u}}{\partial t}+\mathbf{u} \cdot \nabla \mathbf{u}=\frac{1}{\rho_{\mathrm{f}}} \nabla P-\frac{3 \pi \mu n d}{\rho_{\mathrm{f}}}(\mathbf{u}-\mathbf{v})+v \nabla^{2} \mathbf{u}, \\
& \frac{\partial \epsilon}{\partial t}+\nabla \cdot(\epsilon \mathbf{v})=0 \\
& \frac{\partial \mathbf{v}}{\partial t}+\mathbf{v} \cdot \nabla \mathbf{v}=\frac{18 \mu}{\rho_{\mathrm{p}} d^{2}}(\mathbf{u}-\mathbf{v})
\end{aligned}
$$

where $\mathbf{u} \equiv\left(u_{x}, u_{y}\right)$ is the gas velocity, $\mathbf{v} \equiv\left(v_{x}, v_{y}\right)$ is the particle velocity, $P$ is the gas pressure, $\rho_{\mathrm{f}}$ is the gas density. The momentum coupling is described by the term $3 \pi \mu \mathrm{nd} / \rho_{\mathrm{f}}(\mathbf{u}-\mathbf{v})$, where $n$ is the particle number density and $d$ is the particle diameter, $\mu$ and $v$ are the gas dynamic and kinematic viscosity, respectively. $\epsilon$ denotes the particulate local volume fraction (i $\epsilon=n \pi d^{3} / 6$ ). The above mathematical formulation is identical to that given in Saffman's (1962) pioneering paper.

The governing equations are now non-dimensionalized using half the velocity difference across the mixing layer $U_{0}$, the vorticity thickness of the initial mean flow $\delta \equiv 2 U_{0} / \mathrm{d} U / \mathrm{d} y \mid y=0$ and a reference particle volume fraction $\epsilon_{0}$. The resulting equations for the gas and particulate phase become:

$$
\begin{aligned}
& \nabla \cdot \mathbf{u}=0 \\
& \frac{\partial \mathbf{u}}{\partial t}=\mathbf{u} \times \vec{\omega}-\nabla\left(P+|\mathbf{u}|^{2} / 2\right)+\frac{\nabla^{2} \mathbf{u}}{R e}-Z \epsilon \frac{\mathbf{u}-\mathbf{v}}{S t}, \\
& \frac{\partial \epsilon}{\partial t}+\nabla \cdot(\epsilon \mathbf{v})=0
\end{aligned}
$$




$$
\frac{\partial \mathbf{v}}{\partial t}+\mathbf{v} \cdot \nabla \mathbf{v}=\frac{[\mathbf{u}-\mathbf{v}]}{S t}
$$

where $\omega \rightarrow \equiv \nabla \times \mathbf{u}$ is the fluid vorticity. The same symbols are used here to denote the corresponding non-dimensional variables. The flow Reynolds number, $R e$, is defined as $U_{0} \delta / v$. The flow momentum coupling term becomes $Z \epsilon \mathbf{u}-\mathbf{v} / S t$, where $Z$ is the average particle mass loading $\left(Z=\rho_{\mathrm{p}} / \rho_{\mathrm{f}} \epsilon_{0}\right)$; the Stokes number $S t$ represents the ratio of particle response time $\left(\tau_{\mathrm{p}}=d^{2} / 18 v \rho_{\mathrm{p}} / \rho_{\mathrm{f}}\right)$ to flow characteristic time $\left(\tau_{f}=\delta / U_{0}\right)$.

The dimensionless mean velocity profile of the gas phase is assumed to be:

$$
U(y)=\operatorname{erf}(\sqrt{\pi} y)
$$

This velocity profile gives a compact vorticity layer (i.e. vorticity decays very quickly to zero at large y) and was used in previous studies of single-phase mixing layer (e.g. Corcos and Sherman 1984, Moser and Rogers 1993).

Particles are initially distributed uniformly throughout the flow $[\epsilon(t=0)=1]$ with the initial mean velocity equal to the fluid mean velocity. This assumption of dynamic equilibrium in the mean is used in the previous studies by Saffman (1962) and Yang et al. (1990). The perturbation is taken to be periodic in the streamwise $(x)$ direction and is unbounded in the vertical direction $(y)$ with vanishing velocity-vorticity perturbation at infinities. A pseudospectral method was developed to solve the governing equations (Tong and Wang 1997).

We consider the temporal instability of the flow composed of two equal and opposite currents, as in Saffman (1962). This instability mode may be realized in an experimental tilting tank (Thorpe 1968, 1973). We note that, however, most experimental shear layers are composed of two parallel streams moving in the same direction, leading to convectively unstable mode which should be studied as a spatial instability problem (Huerre and Monkewitz, 1985). The relationship between the temporal instability and spatial instability has been discussed, for example, by Michalke (1965). These two problems have qualitatively similar features but are quantitatively different. The observations to be made in this paper, therefore, should only be valid qualitatively when considering a spatially evolving, viscous, particle-laden flow.

The conventional way to obtain the linear instability growth rate of the above twodimensional flow system is to solve a linearized system of Eqs. (6)-(8), or the Orr-Sommerfeld equation. Saffman (1962) showed that the Orr-Sommerfeld equation was similar to that of a single-phase flow, but with the usual mean flow $U(y)$ replaced by a complex mean flow $\bar{u}(y)$

$$
\bar{u}(y)=U(y)+\frac{Z(U-c)}{1+i \alpha(U-c) S t},
$$

where $\alpha$ is the perturbation wave number and $c$ is the phase velocity.

The problem of linear instability is an eigenvalue problem involving four governing parameters, namely, the growth rate, $\sigma r m \equiv \alpha \mathrm{c}_{\mathrm{i}}$, is a function of $\operatorname{Re}, \alpha$, particle inertia parameter $S t$, and particle mass loading $Z$ :

$$
\sigma=\sigma(R e, \alpha, S t, Z)
$$

Here $c_{i}$ is the imaginary part of $c$. As a comparison, in clean gas or single-phase flow, the 
growth rate $\sigma_{0}$ is only a function of $R e$ and $\alpha$ :

$$
\sigma_{0}=\sigma_{0}(R e, \alpha)
$$

The phase velocity or the real part of $c$ is zero due to the symmetry of the mean velocity profile, Eq. (9). It is important to note that the perturbation field in the particle concentration does not enter the eigenvalue problem directly or it can be determined, a posteriori, in terms of the eigenfunctions of the velocity fields.

Saffman (1962) made two important observations regarding the asymptotic solutions in terms of $S t$. For the case of very fine particles or $S t \ll 1$, he used a leading order solution for the particle velocity field to show that the effect of the dust is simply equivalent to increasing the density of gas and thus an increase of flow Reynolds number by a factor of $(1+Z)$. Therefore,

$$
\sigma(\alpha, \operatorname{Re}, Z, S t \ll 1)=\sigma_{0}\left[\alpha, \operatorname{Re}_{\mathrm{e}}=\operatorname{Re}(1+Z)\right] .
$$

Since in a single-phase viscous mixing layer, the growth rate increases with $R e$ (Michalke 1964), the addition of particles then destabilizes the flow. We will compare this relation with numerical results in Section 4.

In the limit of very large particle inertia or $S t \gg 1$, Saffman made another prediction. In this limit, we have $\bar{u}(y)=U(y)-i Z /(\alpha \cdot S t)$ from Eq. (10). Therefore,

$$
\sigma(\alpha, R e, Z, S t \gg 1)=\sigma_{0}(\alpha, R e)-\frac{Z}{S t} .
$$

This relation indicates that the growth rate decreases linearly with loading for large St. Yang et al. (1990) made a similar observation, although with the additional assumption that the flow is inviscid.

We shall now formulate a relation to predict the growth rate at arbitrary St. Basically we shall modify the prediction of Michael (1965) for inviscid two-phase flow. Michael (1965) considered a step velocity profile of $U(y)=1$ for $y>0$ and $U(y)=-1$ for $y<0$. By applying the kinematic (continuity of interface displacement) and dynamic (continuity of the pressure disturbance) conditions at the interface $y=0$, he derived the equation for the growth rate, which in our notation, is

$$
\left(1-\frac{\sigma^{2}}{\alpha^{2}}\right)\left[(1+S t \sigma)^{2}+\alpha^{2} S t^{2}+Z(1+\sigma S t)\right]-2 S t Z \sigma=0
$$

We note that in the limit of $S t \gg 1$ the leading-order solution to Eq. (15) is

$$
\sigma=\alpha-2 \frac{Z}{S t}
$$

this is different from the correct behavior given by Saffman, Eq. (14). This discrepancy is due to the assumption in Michael (1965) that particles do not cross the interface between the gas streams or the normal velocity of the particles relative to this interface is neglected. This assumption is only valid in the limit $S t \ll 1$. 
Eq. (15) has to be solved numerically, in general, to determine the growth rate at the inviscid limit. However, a useful explicit solution for small loading is given by Michael (1965) as

$$
\sigma=\alpha\left[1-\frac{\alpha \cdot Z \cdot S t}{1+2 \alpha \cdot S t+2(\alpha \cdot S t)^{2}}\right]
$$

The first term, $\alpha$, in Eq. (17) represents the growth rate for an inviscid single-phase flow with a step mean velocity, while the second term gives the damping effect of the particulate phase on the instability growth. Assuming that the two parts are separable for a general mean velocity profile, we may approximate the growth rate for an inviscid particle-laden flow as

$$
\sigma(\alpha, Z, S t)=\sigma_{0}(\alpha)-\frac{2 \alpha^{2} \cdot Z \cdot S t}{1+2 \alpha \cdot S t+2(\alpha \cdot S t)^{2}}
$$

where we have simply added a factor 2 to the second term to ensure the correct limiting behavior, Eq. (14). Such correction would have been made more rigorously if we could treat the two interfaces (fluid material interface and the particle material interface) separately when they do not overlap. However, we do not know how to handle analytically boundary conditions at the two separate interfaces. Eq. (18) is expected to predict the growth rate well at large flow Reynolds number and small wavenumber, as the differences between the step velocity profile analyzed by Michael (1965) and a smoothed velocity profile vanish.

We note further that Tatsumi et al. (1964) derived an asymptotic solution for small $\alpha$ of an inviscid, single-phase mixing layer with a hyperbolic tangent velocity profile. Since the solution is insensitive to the shape of the velocity profile for small $\alpha$ and the mean velocity profile used in this study is very similar to the hyperbolic tangent velocity profile, we can write Eq. (19) as

$$
\sigma(\alpha, Z, S t)=\alpha\left[1-1.78 \alpha+3.32 \alpha^{2}\right]-\frac{2 \alpha^{2} \cdot Z \cdot S t}{1+2 \alpha \cdot S t+2(\alpha \cdot S t)^{2}}
$$

We stress that this proposed relation was not derived rigorously and thus its usefulness can only be justified by comparison with numerical results, as will be done in Section 4.3. Although the above relation was developed for small $\alpha$ and $Z$, the comparison with numerical results will show that the relation works well for $\alpha<0.4$ and $Z<1$.

We may go one step further by assuming that the viscous effect can be included in the first term in a manner which can reproduce the limiting behavior at $S t \ll 1$. A more general relation for the growth rate in viscous, particle-laden flow is then

$$
\sigma(\alpha, R e, Z, S t)=\sigma_{0}\left(\alpha, R e_{\mathrm{e}}\right)-\frac{2 \alpha^{2} \cdot Z \cdot S t}{1+2 \alpha \cdot S t+2(\alpha \cdot S t)^{2}}
$$

where $\operatorname{Re}_{e} \equiv \operatorname{Re}[1+Z \exp (-S t)]$. This expression satisfies both limiting behaviors given by Saffman, although the solution for the corresponding single-phase flow has to be known. 


\section{Numerical method}

The Orr-Sommerfeld equation has to be solved numerically to obtain the growth rate of linear instability modes. Alternatively the pseudo-spectral code for the full nonlinear equations (Tong and Wang 1997) can be used to calculate the eigenfunctions and growth rate, which will be explained next.

First, we decompose the velocity and particle volumetric concentration fields into the mean and disturbance fields:

$$
\begin{aligned}
& \mathbf{u}=\left(\begin{array}{c}
U(y) \\
0
\end{array}\right)+A(t)\left(\begin{array}{l}
\hat{u}_{x \mathrm{R}}(y)+i \hat{u}_{x \mathrm{I}}(y) \\
\hat{u}_{y \mathrm{R}}(y)+i \hat{u}_{y \mathrm{I}}(y)
\end{array}\right) \mathrm{e}^{\mathrm{i} \alpha x}, \\
& \mathbf{v}=\left(\begin{array}{c}
U(y) \\
0
\end{array}\right)+A(t)\left(\begin{array}{c}
\hat{v}_{x \mathrm{R}}(y)+i \hat{v}_{x \mathrm{I}}(y) \\
\hat{v}_{y \mathrm{R}}(y)+i \hat{v}_{y \mathrm{I}}(y)
\end{array}\right) \mathrm{e}^{\mathrm{i} \alpha x}, \\
& \epsilon=1+A(t)\left(\hat{\epsilon}_{\mathrm{R}}(y)+i \hat{\epsilon}_{\mathrm{I}}(y)\right) \mathrm{e}^{\mathrm{i} \alpha x},
\end{aligned}
$$

where subscript $R$ denotes the real part and $I$ the imaginary part of eigenfunctions; subscript $x$, $y$ represent velocity components in these two directions, respectively.

Next, initial guess of eigenfunctions has to be made, which are usually chosen to guarantee relatively fast convergence. In the current simulations, the following initial forms were used:

$$
\begin{aligned}
& \hat{u}_{y \mathrm{I}}=\hat{v}_{y \mathrm{I}}=-\frac{1}{(\cosh (\alpha y))^{2}}, \\
& \hat{u}_{y \mathrm{R}}=\hat{v}_{y \mathrm{R}}=\frac{\sinh (\alpha y)}{(\cosh (\alpha y))^{2}}, \\
& \hat{\epsilon}_{\mathrm{R}}=\hat{\epsilon}_{\mathrm{I}}=0 .
\end{aligned}
$$

The initial forms for $\hat{u}_{x \mathrm{R}}, \hat{u}_{x \mathrm{I}}, \hat{v}_{x \mathrm{R}}, \hat{v}_{x \mathrm{I}}$ were obtained by applying the divergence-free condition:

$$
\begin{aligned}
& \hat{u}_{x \mathrm{R}}=\hat{v}_{x \mathrm{R}}=-\frac{\mathrm{d} u_{y \mathrm{I}} / \mathrm{d} y}{\alpha}, \\
& \hat{u}_{x \mathrm{I}}=\hat{v}_{x \mathrm{I}}=\frac{\mathrm{d} u_{y \mathrm{R}} / \mathrm{d} y}{\alpha} .
\end{aligned}
$$

These initial forms along with the decomposition specify the initial flow field. The flow is then advanced in time using a second-order Adams-Bashforth scheme on nonlinear terms and a second-order Crank-Nicholson scheme on linear viscous terms. Perturbation amplitude $A(t)$ is defined such that $\hat{u}_{y \mathrm{I}}(y=0)=1$, and is kept to be less than $1 \%$ throughout the simulations 
to ensure a linear instability behavior. Namely, we started each iteration with $A=0.001$ and the simulation was stopped before $A$ reached 0.01 . The eigenfunctions at the end of the simulation were saved and used to specify the initial perturbation fields for the next iteration. It is necessary to keep $A$ sufficiently small since we used a nonlinear flow code. This iteration procedure continues until all the eigenfunctions converge and the amplitude $A(t)$ varies exponentially with time.

We note that, because of the symmetry in the mean velocity profile, the real parts of velocity eigenfunctions are odd functions and the imaginary parts are even ones (for both the gas phase and particulate phase). The real part of particle volumetric concentration eigenfunction is even and the imaginary part is odd.

At the linear instability stage of the flow evolution, the exponential growth rate of the perturbation fields was computed as

$$
\sigma(t)=\frac{\ln [A(\Delta t) / A(t=0)]}{\Delta t},
$$

where $A(\Delta t)$ is the amplitude at time $\Delta t$. The growth rate will remain constant once eigenfunctions converge. Fig. 1 shows, on a log-linear plot, the time development of the perturbation amplitude at the converged stage under different mass loadings. The slope of each straight line represents the corresponding exponential growth rate.

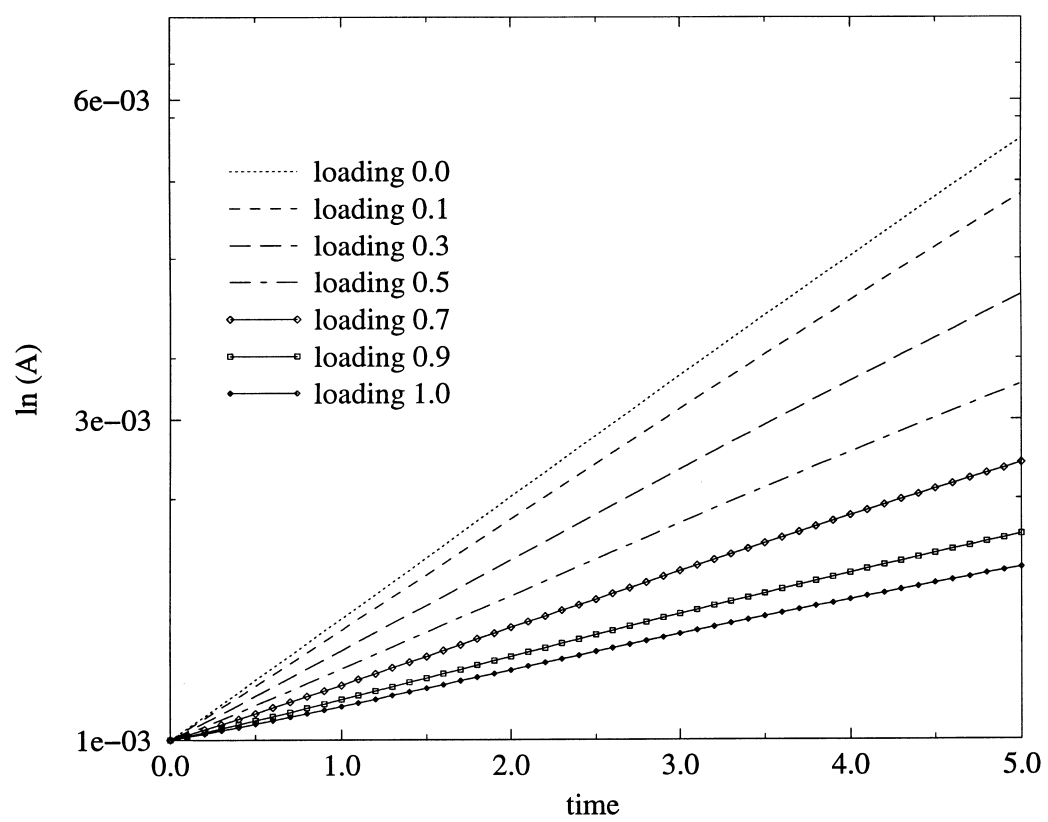

Fig. 1. Time development of perturbation amplitude at the converged stage $(\alpha=0.8620, R e=250, S t=1.0)$. 


\section{Results}

\subsection{Numerical results}

We first present numerical results with each of the three parameters, $\alpha$, St and $Z$, being varied. The Reynolds number is fixed at 250 unless otherwise specified. Based on the singlephase result of Michalke (1964), we expect that the growth rate increases with the Reynolds number. Specifically, the growth rate is sensitive to the Reynolds number only for $R e<500$. For higher Reynolds numbers, the flow is similar to the inviscid limit. Since we intended to show the stabilization effect which is a finite- $R e$ effect, the choice of $R e=250$ was made. Similar results would be expected for other flow Reynolds numbers.

Fig. 2 shows the growth rate as a function of the particle mass loading for three different Stokes numbers and $\alpha=0.8620$. First, for very large Stokes number $(S t=10), \sigma$ decreases linearly with increasing $Z$ and the slope is about $1 / S t$, as predicted by Saffman (1962). The dependence on loading is no longer linear for small to intermediate Stokes numbers. For most Stokes numbers, the growth rate is less than that of a single-phase flow implying that the addition of particles tends to stabilize the flow. However, for very small Stokes number $(S t=0.01)$, the growth rate can actually be larger than that of single phase flow and increases slowly with $Z$ or particles can actually destabilize the flow (even in the absence of the gravity), again consistent with Saffman's prediction. A direct comparison of the numerical results with Saffman's asymptotic limits will be given later.



Fig. 2. Growth rate as a function of mass loading for different Stokes numbers $(\alpha=0.8620, R e=250)$. 


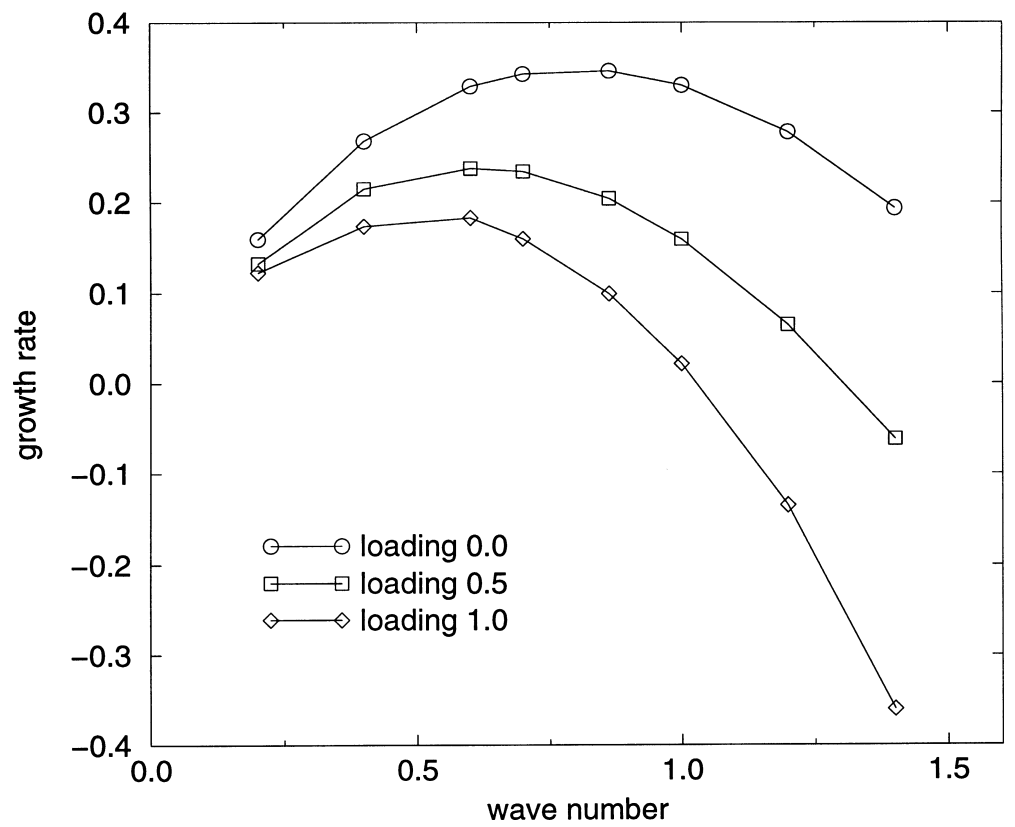

Fig. 3. Growth rate as a function of the wave number under different mass loadings $(R e=250, S t=1.0)$.

Fig. 2 also shows that for a given $\alpha$ and $Z$, the flow becomes most stable for $S t$ of the order of 1 . An interesting implication of this is that particles with intermediate Stokes number may not have the largest dispersion at the later times, unlike the case of one-way coupled particleladen flow.

The growth rate is plotted as a function of $\alpha$ for $S t=1$ in Fig. 3. At this Stokes number, the addition of particles always enhances the stability of the flow and this stabilizing effect increases with the mass loading. Similar to the single-phase case, the growth rate does not depend on $\alpha$ monotonically but rather there is a most unstable wavenumber for each mass loading. This most unstable wavenumber decreases with mass loading, indicating that heavy loading will lead to rollup at longer wavelengths.

Fig. 4 shows the growth rate as a function of the wavenumber with a fixed mass loading $(Z=1.0)$, but different particle inertial parameters. Again we find that particles with Stokes number on the order of unity have the strongest stabilization effect on the flow. This point is demonstrated directly by replotting the same data in Fig. 5 as a function of $S t$.

It should be noted that the linear instability eigenfunctions allow for a qualitative description of the particle-flow interaction, as shown in Fig. 6 in which the perturbed gas vorticity field, the particle velocity divergence and perturbation concentration fields are presented. The particulate velocity field is not divergence free, but has a positive divergence in the vortex center and negative divergence in the braid regions, leading to the depletion of particles in the core and accumulation of particles in the braid regions. This preferential accumulation is known for one-way coupled flow, but appears to be qualitatively similar in the two-way coupled case. The level of accumulation is expected to be reduced in the two-way coupled case as the growth rate is significantly reduced for $S t$ of the order of 1 . 


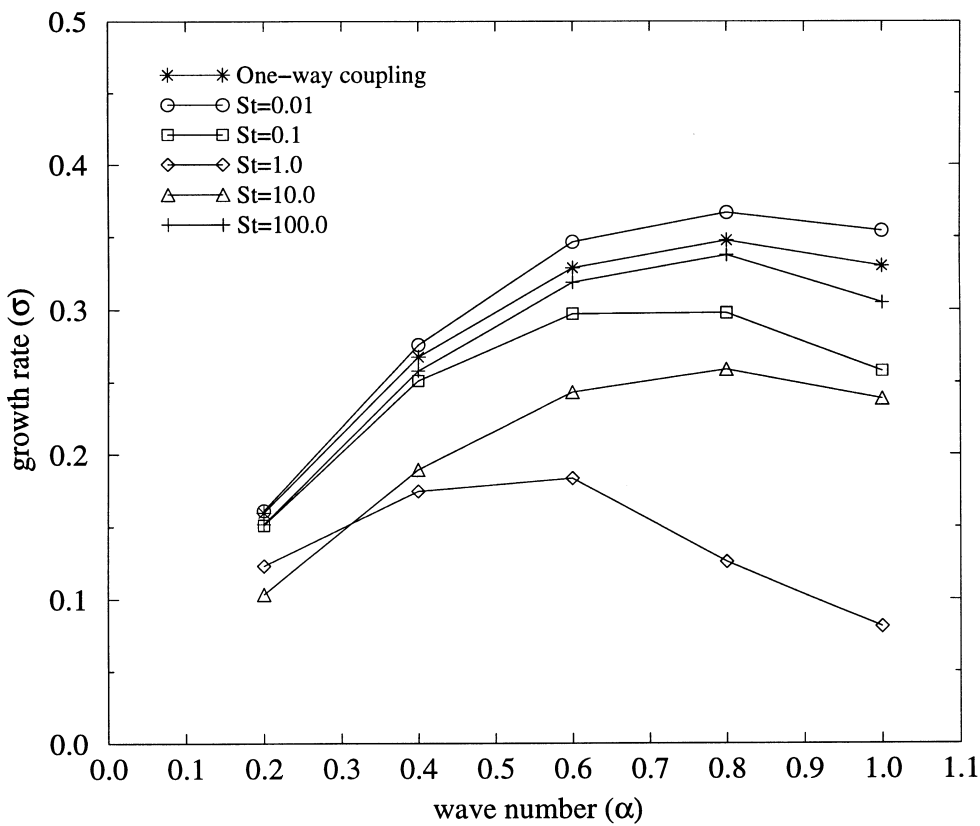

Fig. 4. Growth rate as a function of the wave number for different Stokes numbers $(R e=250, Z=1.0)$.

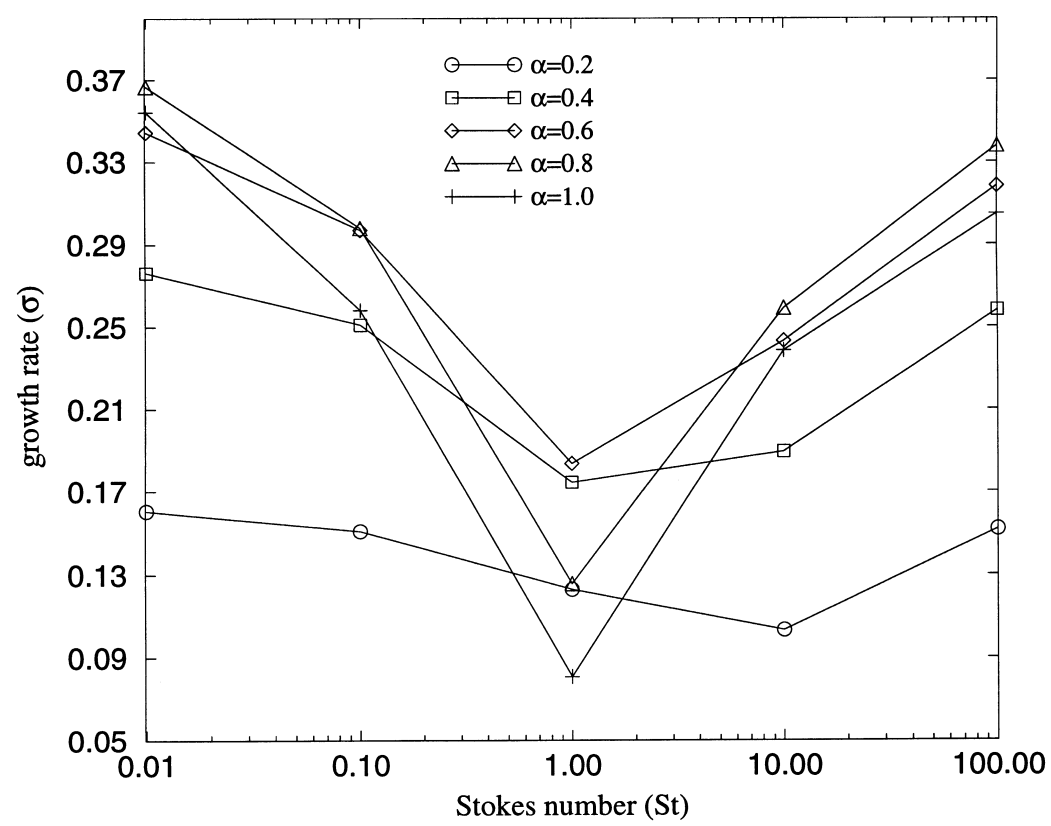

Fig. 5. Growth rate as a function of Stokes number at different wave numbers $(\operatorname{Re}=250, Z=1.0)$. 
(a)

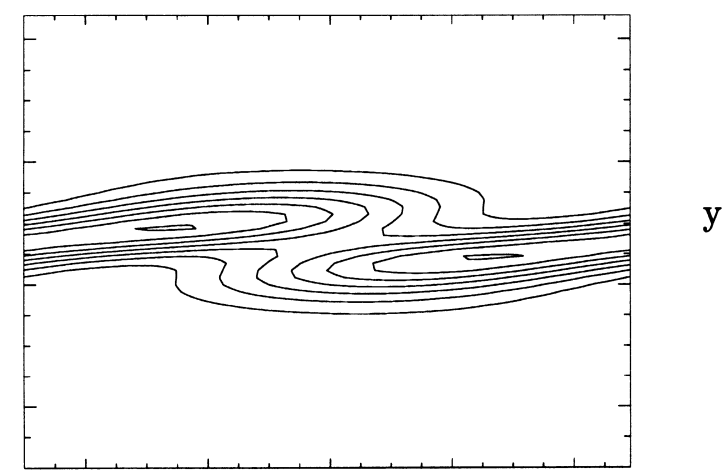

(b)
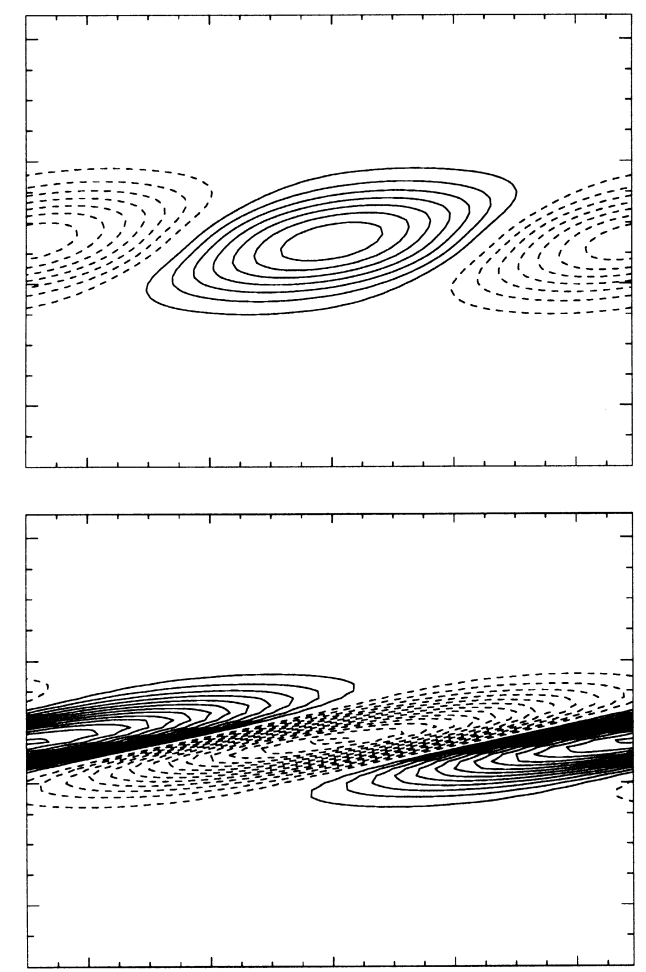

$\mathrm{x}$

Fig. 6. Visualizations of: (a) gas vorticity; (b) particle velocity divergence; and (c) perturbation concentration fields with $\alpha=0.8620, R e=250, S t=1.0$ and $Z=1$. The initial perturbation amplitude is set to 0.08 and contour levels of (a), (b) and (c) are $0.4,0.05$ and 0.1 , respectively.

\subsection{Comparison with Saffman's asymptotic results}

In Fig. 7 we compare the numerical results at large $S t$ with Saffman's asymptotic prediction, Eq. (14). The agreement is well established when $S t$ is on the order of 100 . We also find the agreement is better for large $\alpha$ as the flow time scale based on the wavelength is decreased. 


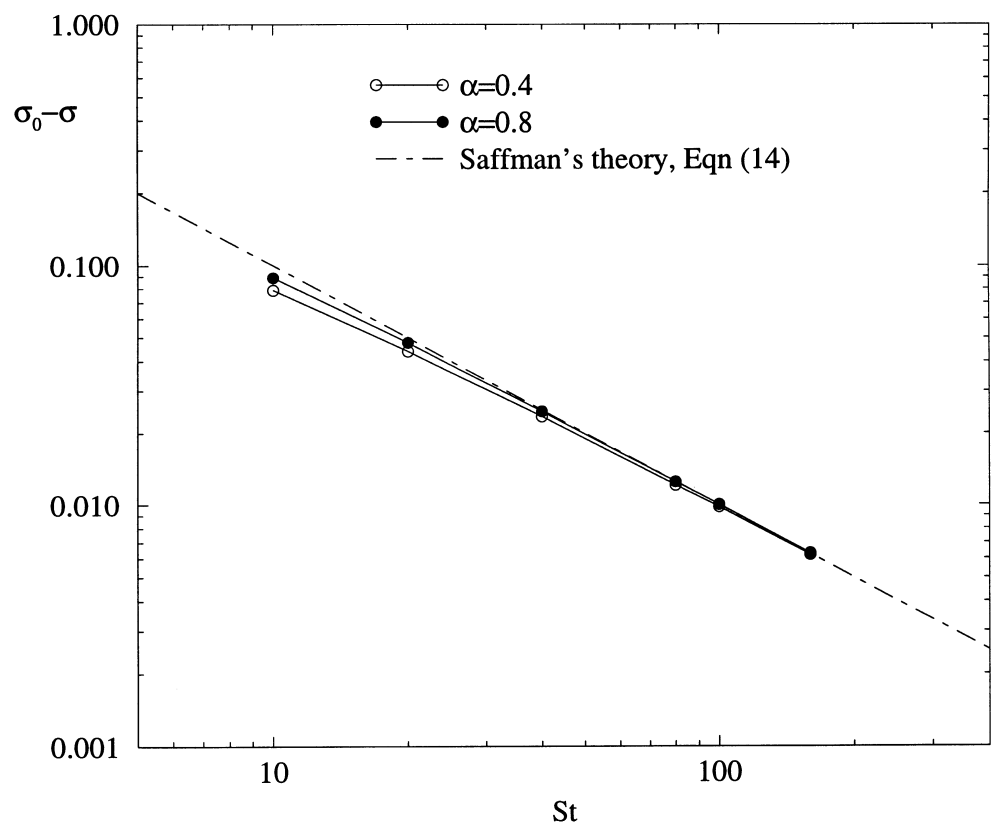

Fig. 7. Comparison of the simulation results with Saffman's theory at the large Stokes number limit $(R e=250$, $Z=1.0)$.

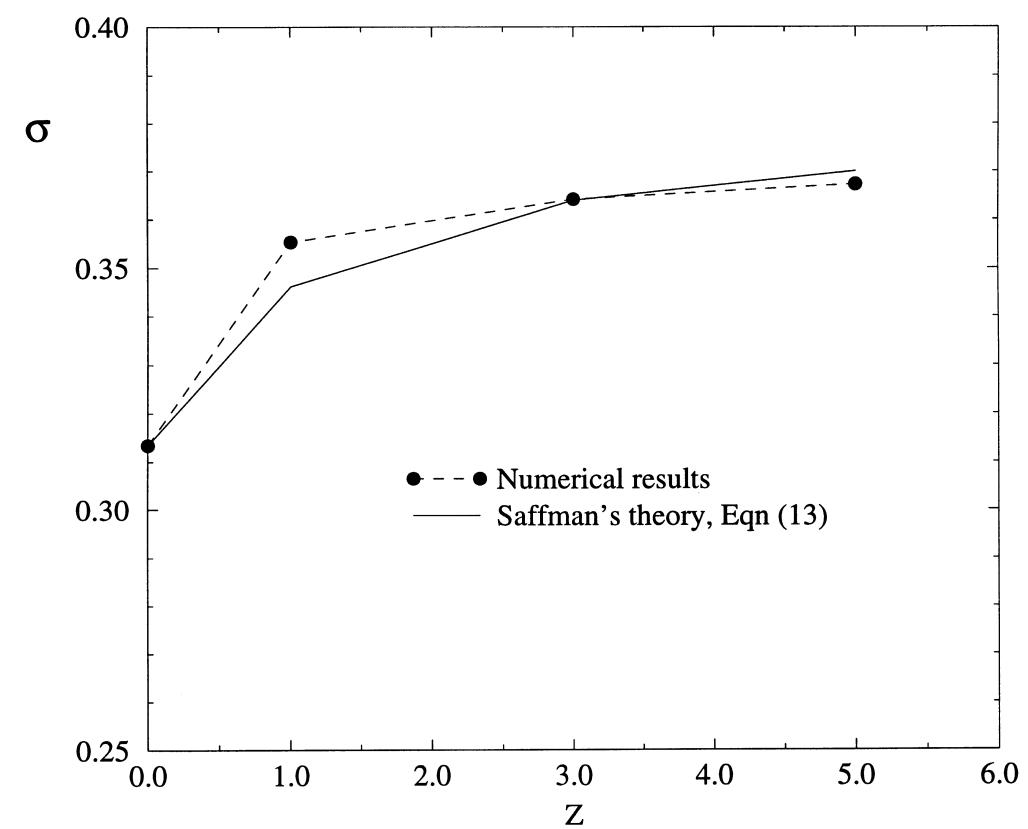

Fig. 8. Comparison of the numerical results with Saffman's theory at the small Stokes number limit $(S t=0.01)$ with $R e=125$ and $\alpha=0.8620$. 


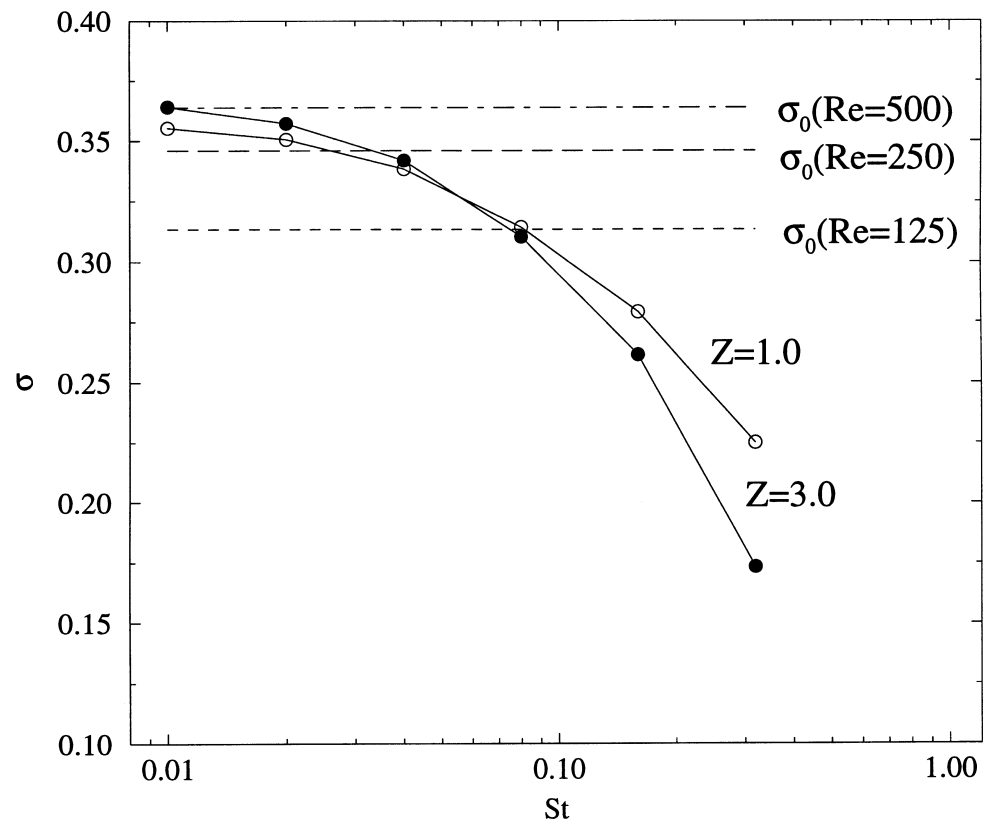

Fig. 9. Comparison of the numerical results with Saffman's theory at the small Stokes number limit $(\alpha=0.8620$ and $R e=125)$. Horizontal lines indicate the growth rate levels of single-phase flow.

Fig. 8 shows a comparison at the small $S t$ limit $(S t=0.01)$. It is harder to obtain accurate numerical growth rates since a smaller time step is necessary. Nevertheless, the destabilizing effect is confirmed and follows approximately the predicted dependence on the mass loading, Eq. (13).

An interesting question is when the destabilizing effect due to the increase of effective inertia of the fluid-particle mixture balances, in terms of $S t$ number, the stabilization effect due to enhanced viscous dissipation around particles. The cross-over of the curves with respect to the $\sigma_{0}(R e=125)$ horizontal line in Fig. 9 shows that particles with $S t<0.08$ show destabilizing effect while particles with $S t>0.08$ give stabilizing influence, at least for loading on the order of one. The three horizontal lines in Fig. 9 denote, respectively, the single-phase flow growth rate, and the predictions at $S t \ll 1$ by Eq. (13) for the two mass loadings considered.

\subsection{Comparison with the proposed relation}

Fig. 10 compares the asymptotic result of Tatsumi et al. (1964) for small $\alpha$ in single-phase flow with numerical results at $R e=250$ and $Z=0$. As expected, the agreement is good for small $\alpha$, say, $\alpha<0.3$.

Fig. 11 provides the comparison of the dependence of the growth rate on $Z$ between simulation results and the proposed relation, Eq. (19), under the conditions of low wave numbers $(\alpha=0.1,0.2)$ and small mass loading $(Z=0.1-0.5)$. Overall the agreement is good, particularly in terms of the slopes of the curves. The numerical results agree well with the 


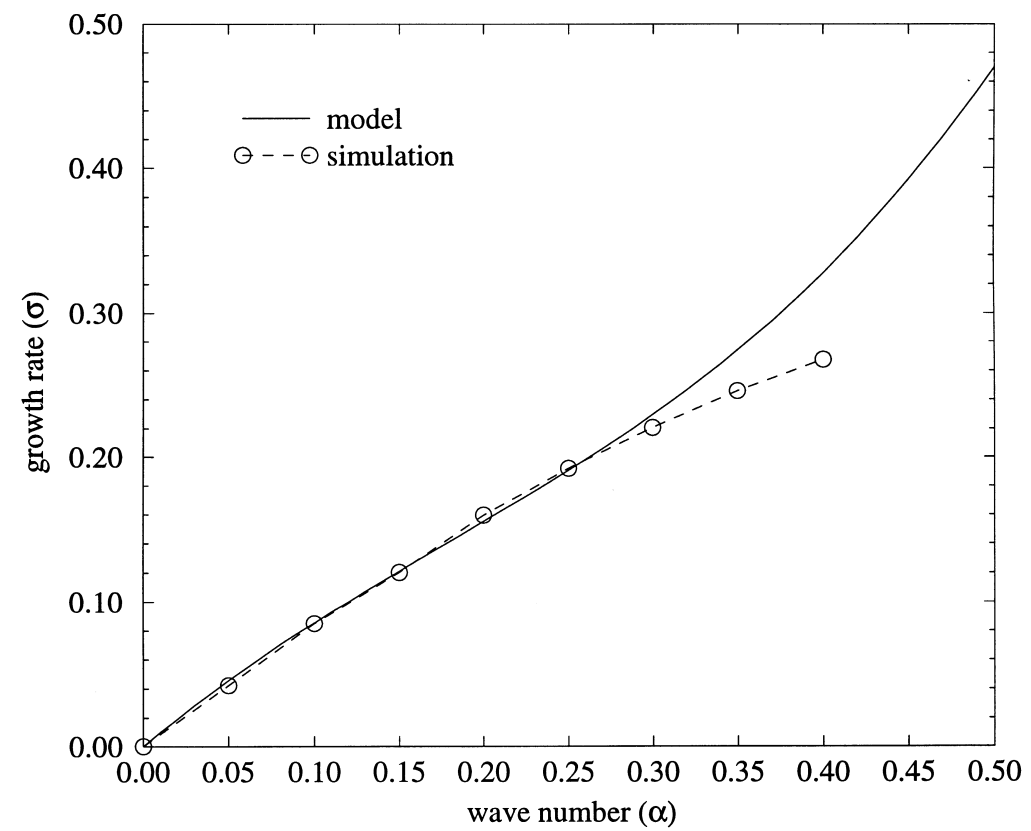

Fig. 10. Comparison of the growth rate as a function of the wave number between the simulation and the model in single-phase mixing layer.

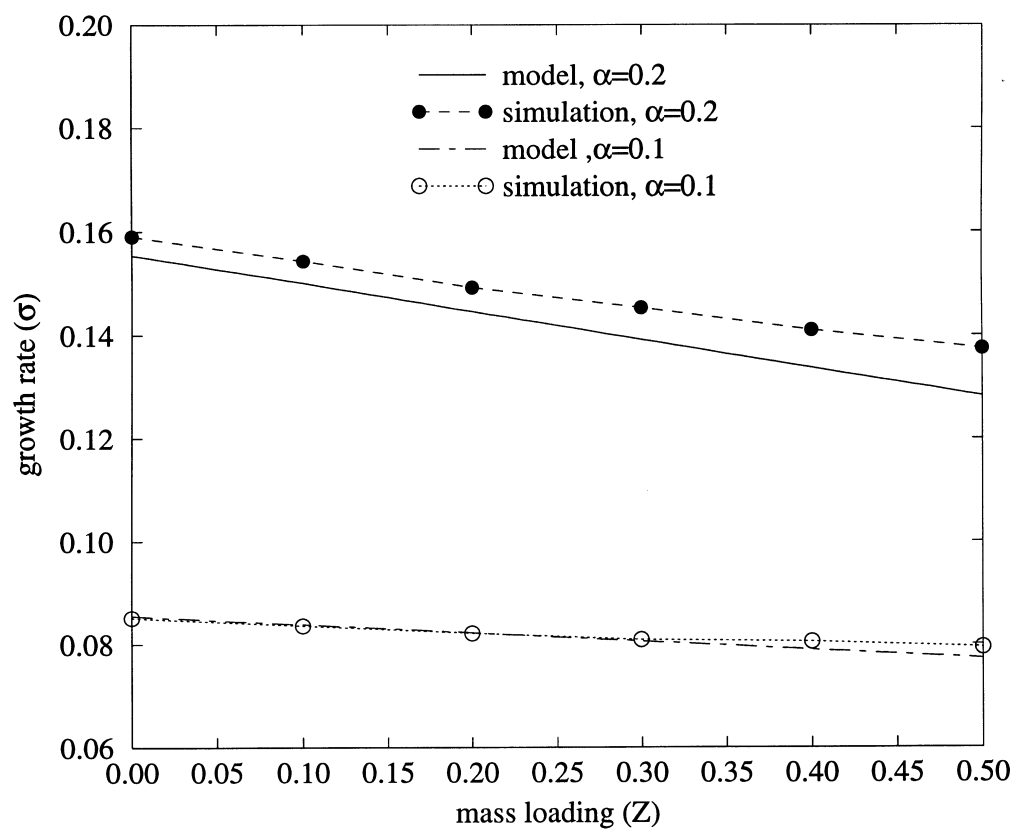

Fig. 11. Comparison of the growth rate as a function of mass loading between the simulation and the model $(R e=250, S t=1.0)$. 


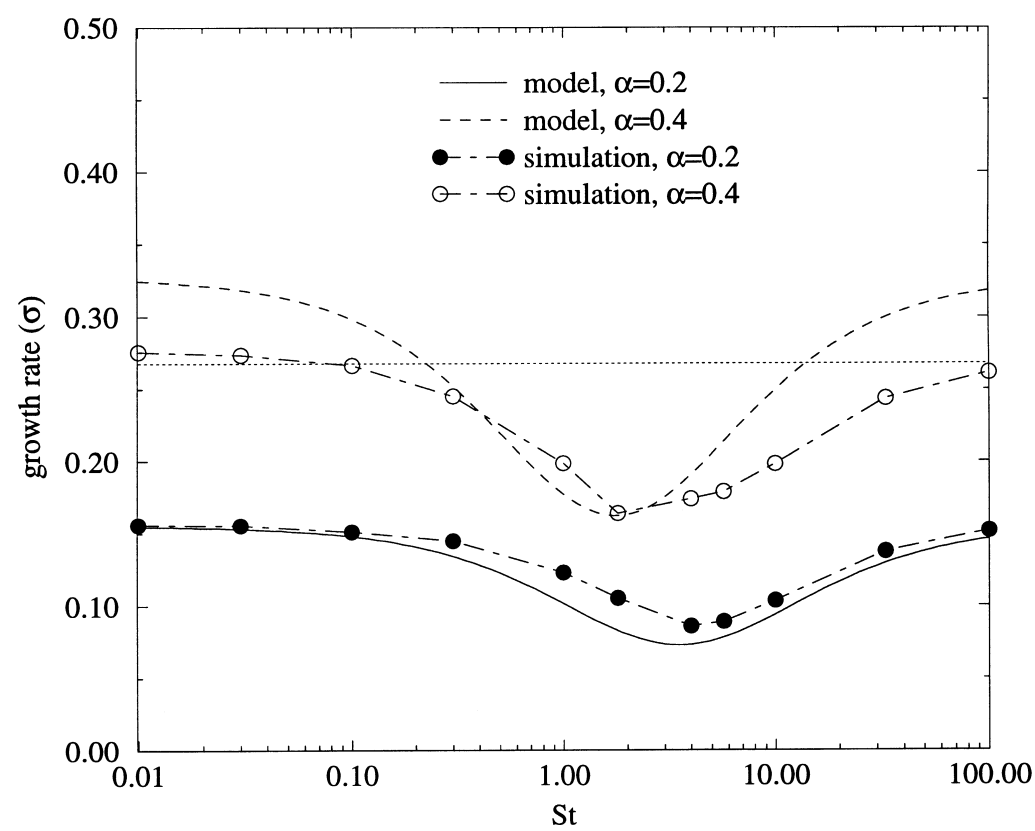

Fig. 12. Comparison of the growth rate as a function of Stokes number between the simulation and the model $(R e=250, Z=1.0)$. The horizontal dotted line denotes the growth rate of single-phase mixing layer at $\operatorname{Re}=250$ and $\alpha=0.4$.

proposed relation. It would have been better to make a comparison with the viscous model if the growth rate at any Reynolds number for the single-phase flow was available.

Fig. 12 compares the inertial dependence of the growth rate. Interestingly the model also predicts that the flow is most stable for intermediate St. In fact, Eq. (19) shows that the Stokes number corresponding to the minimum $\sigma$ is $S t=1 /(\sqrt{ } 2 \alpha$. This result implies that the most damped Stokes number shifts to smaller value as we increase $\alpha$, which is in qualitative agreement with the numerical results. For example, the most damped mode occurs around $S t=4.0$ and $S t=1.8$ for $\alpha=0.2$ and $\alpha=0.4$, respectively, in the simulations.

\subsection{Alternative explanations in terms of energy transfer}

Physically, the addition of particles increases the effective inertia of the fluid-particle mixture, since the local effective density of the mixture is

$$
\rho_{\mathrm{m}}=\rho_{\mathrm{f}}+\rho_{\mathrm{p}} \epsilon \epsilon_{0}=\rho_{\mathrm{f}}(1+Z \epsilon),
$$

and the bulk average mixture density is

$$
\left\langle\rho_{\mathrm{m}}\right\rangle=\rho_{\mathrm{f}}(1+Z) .
$$

Namely the bulk density is increased by a factor of $(1+Z)$. We note that in Eqs. (30) and (31), $\epsilon$ is the non-dimensional volume fraction, $\rho_{\mathrm{f}}$ and $\rho_{\mathrm{m}}$ have the usual dimension of density. For a dilute, Stokes suspension of spherical particles, the increase in the dynamic bulk viscosity 


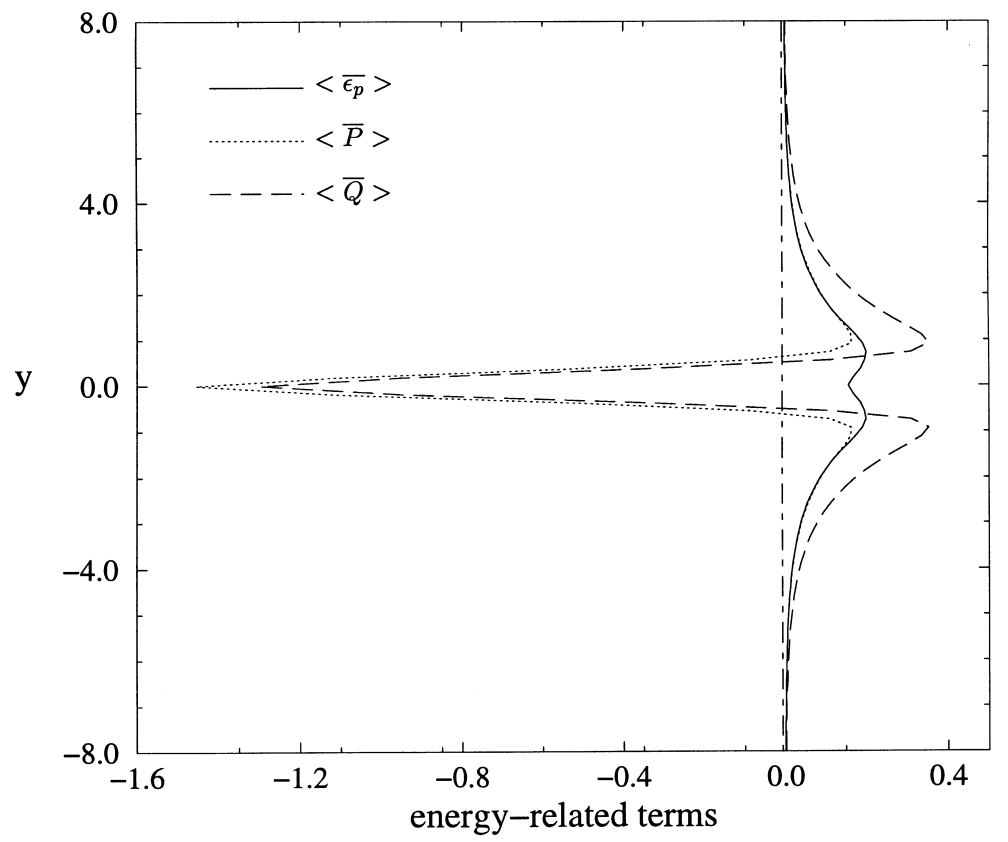

Fig. 13. The distribution of streamwise-averaged energy-related terms for $R e=250, \alpha=0.4, Z=1$, and $S t=1$.

is $5 / 2 \mu \epsilon_{0}$ (see, e.g. Batchelor 1967), which is negligible for a dilute flow considered in this work. Therefore, the increase in the effective inertia leads to an increase in the effective flow Reynolds number, and is thus a destabilization effect. On the other hand, the addition of particles generates extra viscous dissipation as a result of the local flows around particles. This additional dissipation or particle dissipation $\epsilon_{\mathrm{p}}$ per unit volume is

$$
\epsilon_{\mathrm{p}}=\frac{\rho_{\mathrm{f}} U_{0}^{3}}{\delta} Z \epsilon \frac{(\mathbf{u}-\mathbf{v})^{2}}{S t},
$$

where on the right-hand side of Eq. (32), $\rho_{\mathrm{f}}, U_{0}$, and $\delta$ are dimensional quantities and all others are dimensionless. Note that because of the relative motion between the phases, energy is typically transferred from the gas to the particulate phase (but not always in this direction, as shown below). The rate of work transferred to/from the particulate phase is

$$
P=\frac{\rho_{\mathrm{f}} U_{0}^{3}}{\delta} Z \epsilon \frac{\mathbf{v} \cdot(\mathbf{u}-\mathbf{v})}{S t},
$$

while the rate of work transferred from/to the gas is

$$
Q=\frac{\rho_{\mathrm{f}} U_{0}^{3}}{\delta} Z \epsilon \frac{\mathbf{u} \cdot(\mathbf{u}-\mathbf{v})}{S t} .
$$

The local dissipation rate $\epsilon_{\mathrm{p}}$ represents the difference, $Q-P$.

For the case of small $S t$, the relative velocity is on the order of $S t \mathrm{~d} \mathbf{u} / \mathrm{d} t$ (Saffman 1962). Therefore, 


$$
\epsilon_{\mathrm{p}} \approx \frac{\rho_{\mathrm{f}} U_{0}^{3}}{\delta} Z \epsilon S t\left(\frac{\mathrm{d} \mathbf{u}}{\mathrm{d} t}\right)^{2} .
$$

This particle dissipation increases linearly with $S t$ for small $S t$ and leads to stabilization effect. Whether the flow is more stable or not, relative to the single-phase case, depends on the relative magnitudes of the two mechanisms. For a given mass loading, the effective inertia is fixed. In the limit of very small $S t$, the particle dissipation vanishes and thus the flow is less stable. As $S t$ increases, the particle dissipation is increased and thus quickly the flow becomes more stable than the corresponding single-phase flow.

We can compute the above quantities, $\epsilon_{\mathrm{p}}, P$, and $Q$, within the framework of the linear instability. Of interest are the streamwise averages of these quantities, which can be written in terms of the eigenfunctions, using Eqs. (22)-(24). For example,

$$
\begin{aligned}
\frac{\overline{\epsilon_{\mathrm{p}}} \delta}{\rho_{\mathrm{f}} U_{0}^{3}}= & \frac{Z}{S t} \overline{\left(\mathbf{u}^{\prime}-\mathbf{v}^{\prime}\right)^{2}} \\
= & \frac{Z}{S t} \frac{A^{2}(t)}{2}\left[\left(\hat{u}_{x \mathrm{R}}(y)-\hat{v}_{x \mathrm{R}}(y)\right)^{2}\right. \\
& +\left(\hat{u}_{x \mathrm{I}}(y)-\hat{v}_{x \mathrm{I}}(y)\right)^{2} \\
& +\left(\hat{u}_{y \mathrm{R}}(y)-\hat{v}_{y \mathrm{R}}(y)\right)^{2} \\
& \left.+\left(\hat{u}_{y \mathrm{I}}(y)-\hat{v}_{y \mathrm{I}}(y)\right)^{2}\right] \\
\frac{\bar{Q} \delta}{\rho_{\mathrm{f}} U_{0}^{3}=} & \frac{Z}{S t}\left(\overline{\mathbf{u}^{\prime} \cdot\left(\mathbf{u}^{\prime}-\mathbf{v}^{\prime}\right)}+U \overline{\epsilon^{\prime}\left(u_{x}^{\prime}-v_{x}^{\prime}\right)}\right) \\
= & \frac{Z}{S t} \frac{A^{2}(t)}{2}\left[\hat{u}_{x \mathrm{R}}(y)\left(\hat{u}_{x \mathrm{R}}(y)-\hat{v}_{x \mathrm{R}}(y)\right)\right. \\
& +\hat{u}_{x \mathrm{I}}(y)\left(\hat{u}_{x \mathrm{I}}(y)-\hat{v}_{x \mathrm{I}}(y)\right) \\
& +\hat{u}_{y \mathrm{R}}(y)\left(\hat{u}_{y \mathrm{R}}(y)-\hat{v}_{y \mathrm{R}}(y)\right) \\
& +\hat{u}_{y \mathrm{I}}(y)\left(\hat{u}_{y \mathrm{I}}(y)-\hat{v}_{y \mathrm{I}}(y)\right) \\
& +U(y) \hat{\epsilon}_{\mathrm{R}}(y)\left(\hat{u}_{x \mathrm{R}}(y)-\hat{v}_{x \mathrm{R}}(y)\right) \\
& \left.+U(y) \hat{\epsilon}_{\mathrm{I}}(y)\left(\hat{u}_{x \mathrm{I}}(y)-\hat{v}_{x \mathrm{I}}(y)\right)\right],
\end{aligned}
$$

where the overbar denotes the streamwise average and ' denotes the perturbation fields. Fig. 13 shows the distribution of these streamwise-averaged quantities for a typical case at $R e=250$, $\alpha=0.4, Z=1$, and $S t=1$. Interestingly, both $\bar{P}$ and $\bar{Q}$ are negative near the interface of the mixing layer, implying that the kinetic energy is transferred from the particulate phase to the gas. A careful examination of the velocity vector plots reveals the origin of this direction of transfer: the gas perturbation velocity is typically pointing away from the center (i.e. the point which will become vortex center at the later nonlinear rollup) and the relative velocity, $\left(\mathbf{v}^{\prime}-\mathbf{u}^{\prime}\right)$, is also pointing away from the center due to the inertial bias effect (Maxey 1987); this combination leads to larger particulate perturbation velocity near the interface and thus the 
observed direction of transfer. Away from the interface, the direction of transfer is reversed. The dissipation $\left(\overline{\epsilon_{\mathrm{p}}}\right)$ profile shows a peak at a location where the direction of the transfer is reversed.

The profiles can be further integrated over $y$ to compute the bulk energy-related budgets, and the final results are denoted by $\left\langle\overline{\epsilon_{\mathrm{p}}}\right\rangle,\langle\bar{P}\rangle$, and $\langle\bar{Q}\rangle$ :

$$
\begin{aligned}
& \frac{\left\langle\overline{\epsilon_{\mathrm{p}}}\right\rangle \delta}{\rho_{\mathrm{f}} U_{0}^{3}}=\frac{Z}{S t}\left\langle\overline{\left(\mathbf{u}^{\prime}-\mathbf{v}^{\prime}\right)^{2}}\right\rangle \\
& \frac{\langle\bar{P}\rangle \delta}{\rho_{\mathrm{f}} U_{0}^{3}}=\frac{Z}{S t}\left[\left\langle\overline{\mathbf{v}^{\prime} \cdot\left(\mathbf{u}^{\prime}-\mathbf{v}^{\prime}\right)}\right\rangle+\left\langle U \overline{\epsilon^{\prime}\left(u_{x}^{\prime}-v_{x}^{\prime}\right)}\right)\right] \\
& \frac{\langle\bar{Q}\rangle \delta}{\rho_{\mathrm{f}} U_{0}^{3}}=\frac{Z}{S t}\left[\left\langle\overline{\mathbf{u}^{\prime} \cdot\left(\mathbf{u}^{\prime}-\mathbf{v}^{\prime}\right)}\right\rangle+\left\langle U \overline{\epsilon^{\prime}\left(u_{x}^{\prime}-v_{x}^{\prime}\right)}\right\rangle\right] .
\end{aligned}
$$

These integral quantities can be used to give an alternative explanation for the flow instability characteristics discussed earlier. Fig. 14 shows these global budgets as a function of $S t$ for $R e=250, \alpha=0.4$, and $Z=1$. As expected, for a given loading, the particle dissipation increases quickly with $S t$ when $S t$ is small, eventually leading to a more stable flow relative to the single-phase case at intermediate and larger $S t$ values. Another way of looking at the effect of particles on the flow instability is through the sign of the $\langle\bar{Q}\rangle$ term. $\langle\bar{Q}\rangle$ is negative for small $S t$ and positive for large $S t$, implying qualitatively different effects of the addition of the particles on the gas flow. Obviously, the flow is less stable than the single-phase case if $\langle\bar{Q}\rangle<0$

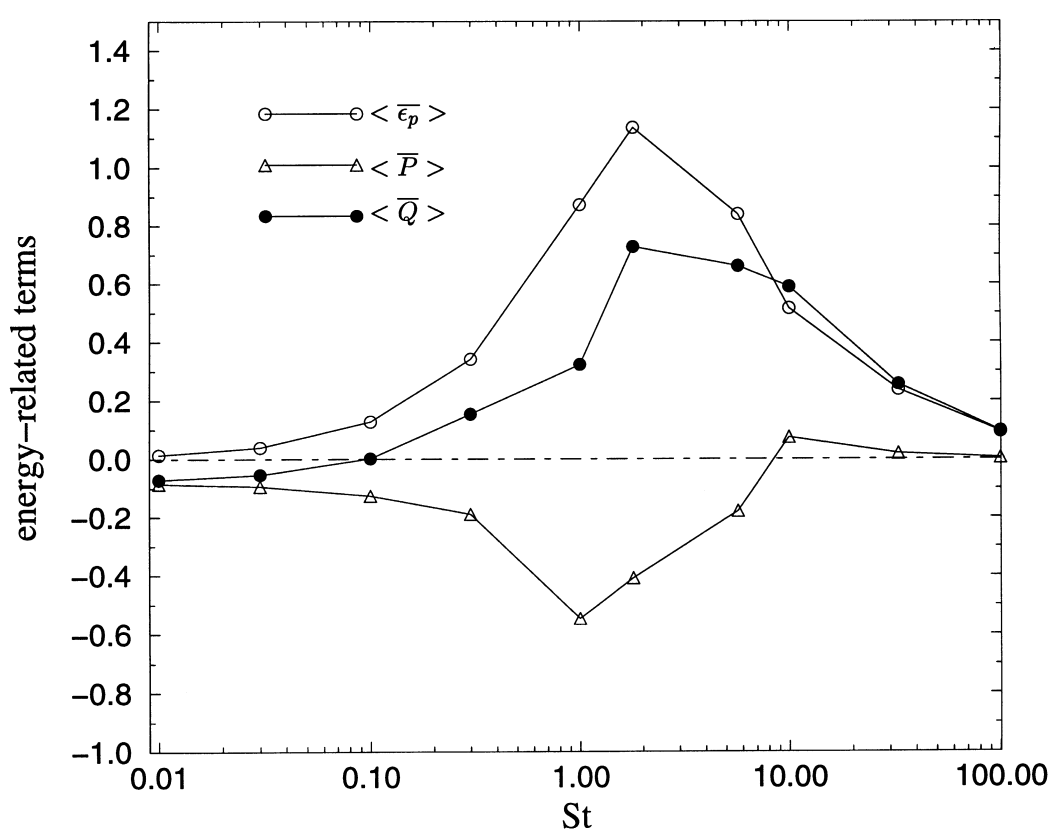

Fig. 14. Energy-related budgets as a function of $\operatorname{St}(\operatorname{Re}=250, \alpha=0.4, Z=1.0)$. 
and more stable if $\langle\bar{Q}\rangle>0$. The transition occurs at $S t \approx 0.10$ for the particular parameter setting. Note this transition is quantitatively the same as that shown in Fig. 10 for $\alpha=0.4$ by the horizontal dotted line.

The limiting behaviors of $\langle\bar{Q}\rangle$ for very large and very small $S t$ can be inferred from an asymptotic analysis similar to that presented in Section 2. Such an asymptotic analysis of the energy transfer budgets can offer insights into the physics of energy transfer characteristics. We first note that the linearization of the volume fraction equation, Eq. (7), implies a scaling for the magnitude of $\epsilon^{\prime}$ as

$$
\epsilon^{\prime} \sim \frac{\nabla \cdot \mathbf{v}^{\prime}}{\sqrt{\sigma^{2}+\alpha^{2} U^{2}}} .
$$

At very large $S t, \epsilon^{\prime}$ is very small because $\mathbf{v}^{\prime}$ is very small, so that the second term in Eq. (40) can be neglected relative to the first term. Also at very small $S t$, the divergence $\nabla \cdot \mathbf{v}^{\prime}$ is linearly proportional to $S t$ (Maxey 1987), so is $\epsilon^{\prime}$. It follows that the second term in Eq. (40) is again much smaller than the first term. Therefore, at large $S t$, the leading order is $(1 / S t)$,

$$
\begin{aligned}
& \left.\frac{\langle\bar{Q}\rangle \delta}{\rho_{\mathrm{f}} U_{0}^{3}}=\frac{\left\langle\overline{\epsilon_{\mathrm{p}}}\right\rangle \delta}{\rho_{\mathrm{f}} U_{0}^{3}}=\frac{Z}{S t} \overline{\left(\mathbf{u}^{\prime}\right)^{2}}\right\rangle, \\
& \frac{\langle\bar{P}\rangle \delta}{\rho_{\mathrm{f}} U_{0}^{3}}=0 .
\end{aligned}
$$

Therefore, the gas phase loses kinetic energy due to the relative motion and this energy is almost completely dissipated locally. At very small $S t$ number, the leading order expansion of the relative velocity leads to

$$
\begin{aligned}
& \frac{\langle\bar{Q}\rangle \delta}{\rho_{\mathrm{f}} U_{0}^{3}} \approx \frac{\langle\bar{P}\rangle \delta}{\rho_{\mathrm{f}} U_{0}^{3}} \\
& \approx Z \overline{\left.\mathbf{u}^{\prime} \cdot \frac{\mathrm{d} \mathbf{u}^{\prime}}{\mathrm{d} t}\right\rangle} \\
& =Z\left[\sigma \overline{\left(\mathbf{u}^{\prime}\right)^{2}}\right\rangle-\left\langle\overline{\left.\left.\left(-u_{x}^{\prime} u_{y}^{\prime}\right) \frac{\mathrm{d} U}{\mathrm{~d} y}\right\rangle\right]}\right. \\
& \approx-\frac{Z}{\operatorname{Re}(1+Z)}\left\langle\overline{\left.\frac{\partial u_{i}^{\prime}}{\partial x_{j}} \frac{\partial u_{i}^{\prime}}{\partial x_{j}}\right\rangle}\right.
\end{aligned}
$$

namely, $\langle\bar{Q}\rangle$ is negative and related to the difference between the rate of increase of gas kinetic energy and the rate of production of the kinetic energy due to the mean shear which is the minus rate of viscous dissipation in the gas perturbation field. Therefore, in this limit, the particulate phase gives energy to the gas phase, qualitatively different from the large St. Note that Eq. (44) follows from the leading-order expansion of the relative velocity, Eq. (45) from 
the linearization of the fluid acceleration, and Eq. (46) from the expanded kinetic energy equation. For the case of $S t=0.01, R e=250, \alpha=0.4$ and $Z=1$, the three ways of computing $\langle\bar{Q}\rangle$, namely, Eqs. (40), (45) and (46), give roughly a same result. These asymptotic results confirm the different signs of $\langle\bar{Q}\rangle$ as shown in Fig. 14.

We note that there were experimental studies on both the kinetic energy transfer to the particulate phase, $\bar{P}$, (Kiger and Lasheras 1995) and additional viscous dissipation due to the particle-fluid interaction, $\overline{\epsilon_{\mathrm{p}}}$, (Kiger and Lasheras 1997) in a nonlinear, turbulent mixing layer with polydispersed, small, spherical particles laden in only half of the mixing layer. Kiger and Lasheras found that the maximum positive value of $\bar{P}$ is located under the core and the maximum negative of it occurs near the free stagnation point mainly due to the large slip velocity between particles and fluid in those regions. In our simulations, although we did not investigate the local distribution of $\bar{P}$, two peaks of $\bar{P}$ shown in Fig. 13 reflect the above results qualitatively to some extent. They also found that the additional viscous dissipation is primarily concentrated into intense regions located beneath the core of the vortex. The same trend is observed by examining the $\overline{\epsilon_{\mathrm{p}}}$ profile in Fig. 13. Their experimental results have also shown that the level of the additional viscous dissipation increases with the particle mass loading for Stokes number of the order of 1, which compares well with our simulation results.

\section{Summary}

We have performed stability analysis of a temporally evolving particle-laden mixing layer, through numerical simulations equivalent to solving the Orr-Sommerfeld equation originally proposed by Saffman (1962). We have confirmed, for the first time, the stability behaviors at the two asymptotic limits, $S t \ll 1$ and $S t \gg 1$, discovered by Saffman (1962). While the stabilizing effect of particles on the flow at large Stokes number is well recognized in the studies by Michael (1965) and Yang et al. (1990) in the inviscid limit, the destabilizing influence at small Stokes number is only realized at finite flow Reynolds number. The fact that the addition of particles can destabilize the flow in the absence of gravity is clearly established here by careful numerical simulations. It should be noted that with the addition of gravity, particles can introduce buoyancy effects which can destabilize the flow easily (Herbolzheimer 1983, Shaqfeh and Acrivos 1986, Borhan and Acrivos 1988). The qualitatively different effects of the addition of the particles on the flow instability have also been explained in terms of the particle dissipation and effective system inertia, as well as the direction of the interphase energy transfer.

We have also provided results on the stability of particle laden mixing layer at arbitrary mass loading, Stokes number, and wavenumber. For a given mass loading and wavenumber, there is an intermediate Stokes number which corresponds to a maximum flow stability. This was speculated by Saffman (1962) based on the two asymptotic limits. We have shown that this Stokes number is on the order of one, and seems to be related to the wavenumber, as implied in the simple model of Michael (1965). An approximate for predicting the growth rate in a general viscous, particle-laden flow is proposed and compared with the simulation results. It was shown that the proposed relation works well for $\alpha<0.4$ and $Z<1$. The efficient damping by the particulate phase at the intermediate Stokes number has an interesting implication that 
the enhanced dispersion discovered previously under one-way coupling (see, e.g. Crowe et al. 1993) might not apply to strongly coupled shear flows. The dispersion of particles in coupled shear flows requires further investigation.

The qualitative correlation between the particle concentration field and the flow vorticity field observed previously under one-way coupling is also shown to exist with two-way coupled dynamics, although the level of particle accumulations will develop at the nonlinear stage of the mixing layer evolutions. This and other related issues of nonlinear two-way coupled mixing layer are studied in a companion paper (Wang et al. 1998a).

\section{Acknowledgements}

We are grateful to Professor Martin Maxey of Brown University for bringing to our attention the founding work by Saffman (1962). Professor Pablo Huq at the University of Delaware provided several constructive comments to this paper. Most of the simulations were performed on the Power Challenge supercomputer at our University. Part of this work is supported by IBM Watson Research Center and the State of Delaware.

\section{References}

Batchelor, G.K., 1967. An Introduction to Fluid Dynamics. Cambridge University Press, Cambridge.

Borhan, A., Acrivos, A., 1988. The sedimentation of nondilute suspensions in inclined settlers. Phys. Fluids 31 (12), 3488-3501.

Corcos, G.M., Sherman, F.S., 1984. The mixing layer: deterministic models of a turbulent flow. Part I. introduction and the two-dimensional flow. J. Fluid Mech. 139, 29-65.

Crowe, C.T., Chung, J.N., Troutt, T.R., 1993. Particle dispersion by organized turbulent structures. In: M.C. Roco (Ed.). Particulate Two-phase Flows, chap. 18. Butterworth-Heinemann, New York.

Crowe, C.T., Sommerfeld, M., Tsuji, Y., 1997. Multiphase Flows with Droplets and Particles. CRC Press, Boca Raton, FL.

Dimas, A.A., Kiger, K.T., 1998. Linear instability of a particle-laden mixing layer with a dynamic dispersed phase. Phys. Fluids 10, 2539-2557.

Elghobashi, S., 1994. On predicting particle-laden turbulent flows. Appl. Sci. Res. 52, 309-329.

Elghobashi, S., Truesdell, G.C., 1993. On the two-way interaction between homogeneous turbulence and dispersed solid particles. I: turbulence modulation. Phys. Fluids 5, 1790-1801.

Gore, R.A., Crowe, C.T., 1991. Modulation of turbulence by a dispersed phase. ASME J. Fluid Engng 113, 304-307. Herbolzheimer, E., 1983. Stability of the flow during sedimentation in inclined channels. Phys. Fluids 26 (8), 2043-2054. Hetsroni, G., 1989. Particle-turbulence interaction. Int. J. Multiphase flow 15, 735-746.

Hishida, K., Ando, A., Maeda, M., 1992. Experiments on particle dispersion in a turbulent mixing layer. Int. J. Multiphase flow 18, 181-194.

Huerre, P., Monkewitz, P.A., 1985. Absolute and convective instabilities in free shear layers. J. Fluid Mech. 159, 151168.

Isakov, E.B., Rudnyak Ya, V., 1995. Stability of rarefied dusty gas and suspension flows in a plane channel. Fluid Dynamics 30, 708-712.

Kiger, K.T., Lasheras, J.C., 1995. The effect of vortex pairing on particle dispersion and kinetic energy transfer in a two-phase turbulent shear layer. J. Fluid Mech. 302, 149-178.

Kiger, K.T., Lasheras, J.C., 1997. Dissipation due to particle/turbulence interaction in a two-phase, turbulent, shear layer. Phys. Fluids 9, 3005-3023. 
Lazaro, B.J., Lasheras, J.C., 1992a. Particle dispersion in the developing shear layer. Part I. unforced flow. J. Fluid Mech. 235, 143-178.

Lazaro, B.J., Lasheras, J.C., 1992b. Particle dispersion in the developing shear layer. Part II. forced flow. J. Fluid Mech. 235, 179-221.

Martin, J.E., Meiburg, E., 1994. The accumulation and dispersion of heavy particles in forced two-dimensional mixing layer. Part 1: the fundamental and two-dimensional cases. Phys. Fluids 6, 1116-1132.

Maxey, M.R., 1987. The gravitational setting of aerosol particles in homogeneous turbulence and random flow fields. J. Fluid Mech. 174, 441-465.

Michael, D.H., 1965. Kelvin-Helmholtz instability of a dusty gas. Proc. Camb. Phil. Soc. 61, 569-571.

Michalke, A., 1964. On the invisid instability of the hyperbolic-tangent velocity profile. J. Fluid Mech. 19, 543-556.

Michalke, A., 1965. On spatially growing disturbances in an inviscid shear layer. J. Fluid Mech. 23, 521-544.

Moser, R.D., Rogers, M.M., 1993. The three-dimensional evolution of a plane mixing layer: pairing and transition to turbulence. J. Fluid Mech. 247, 275-320.

Raju, N., Meiburg, E., 1995. The accumulation and dispersion of heavy particles in forced two-dimensional mixing layers. Part 2: the effect of gravity. Phys. Fluids 7, 1241-1264.

Saffman, P.G., 1962. On the stability of laminar flow of a dusty gas. J. Fluid Mech. 13, 120-128.

Samimy, M., Lele, S.K., 1991. Motion of particles with inertia in a compressible free shear layer. Phys. Fluids A 3, 1915-1923.

Shaqfeh, E.S.G., Acrivos, A., 1986. The effects of inertia on the stability of the convective flow in inclined particle settlers. Phys. Fluids 30 (4), 960-973.

Shirolkar, J.S., Coimbra, C.F.M., Queiroz, McQuay M., 1996. Fundamental aspects of modeling turbulent particle dispersion in dilute flows. Prog. Energy Combust. Sci. 22, 363-399.

Squires, K.D., Eaton, J.K., 1990. Particle response and turbulence modification in isotropic turbulence. Phys. Fluids A 2 (7), 1191-1203.

Sykes, D., Lyell, M.J., 1994. The effect of particle loading on the spatial stability of a circular jet. Phys. Fluids 6, 19371939.

Tatsumi, T., Gotoh, K., Ayukawa, K., 1964. The stability of a free boundary layer at large Reynolds numbers. J. Phys. Soc. Jpn 19 (10), 1966-1980.

Thorpe, S.A., 1968. A method for producing a shear flow in s stratified fluid. J. Fluid Mech. 32, 693-704.

Thorpe, S.A., 1973. Experiments on instability and turbulence in a stratified flow. J. Fluid Mech. 61, 731-751.

Tong, X.L., Wang, L.P., 1997. Direct simulation of three dimensional particle-laden mixing layers. ADVANCES IN DNS/LES. Greyden Press.

Torobin, L.B., Gauvin, W.H., 1961. Fundamental aspects of solids-gas flow, part VI: multiparticle behavior in turbulent fluids. Can. J. Chem. Engng 39, 113-120.

Wang, L.P., 1992. Dispersion of particles injected nonuniformly in a mixing layer. Phys. Fluids A 4, 1599-1601.

Wang, L.P., Tong, X.L., DeSpirito, J., 1998a. Two-way coupled particle-laden mixing layer, Part 2. nonlinear evolution. Presented at the 3rd International Conference on Multiphase Flows. Lyon, France June 8-12, 1998.

Wang, Q.Z., Squires, K.D., Wang, L.P., 1998b. On the effect of nonuniform seeding on particle dispersion in twodimensional mixing layers. Phys. Fluids 10, 1700-1714.

Wen, F., Evans, J., 1994. Linear instability of a two-layer flow with differential particle loading. Phys. Fluids 6, 38933905.

Yang, Y.Q., Chung, J.N., Troutt, T.R., Crowe, C.T., 1990. The influence of particles on the spatial stability of twophase mixing layers. Phys. Fluids 2 (10), 1839-1845.

Yarin, L.P., Hetsroni, G., 1994. Turbulence intensity in dilute two-phase flows-3. The particles-turbulence interaction in dilute two-phase. Int. J. Multiphase flow 20, 27-44.

Yuan, Z., Michaelides, E.E., 1992. Turbulence modulation in particulate flows - a theoretical approach. Int. J. Multiphase flow 18, 779-785. 